\title{
Calponin-h2 is upregulated in the tissues and plasma of patients with breast cancer
}

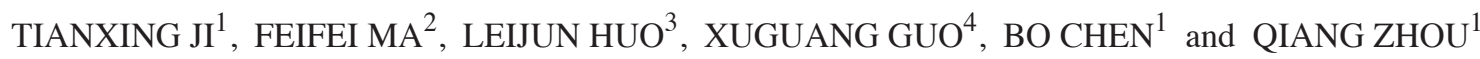 \\ Departments of ${ }^{1}$ Clinical Laboratory and ${ }^{2}$ VIP Obstetrics, The Second Affiliated Hospital of Guangzhou Medical University, \\ Guangzhou, Guangdong 510260; ${ }^{3}$ Department of Pathology, Guangdong Women and Children Hospital, \\ Guangzhou, Guangdong 511442; ${ }^{4}$ Department of Clinical Laboratory, The Third Affiliated Hospital \\ of Guangzhou Medical University, Guangzhou, Guangdong 510140, P.R. China
}

Received August 1, 2014; Accepted April 4, 2015

DOI: $10.3892 / \mathrm{mmr} .2015 .3782$

\begin{abstract}
Increasing evidence has demonstrated that changes in plasma nuclear matrix proteins are specific markers of cancer. Furthermore, proteomic analysis has revealed that calponin-h2 is upregulated in human breast cancer tissue, but is absent in healthy and benign controls. However, the roles of levels of plasma calponin-h2 in the diagnosis of breast cancer and its association with clinicopathological parameters remain to be elucidated. In the present study, the plasma levels of calponin-h2 in patients with breast cancer, benign breast disease and in healthy controls were examined using an enzyme-linked immunosorbent assay. The expression levels of calponin-h2 in invasive breast cancer and normal breast tissues were measured using immunohistochemistry. Statistical analyses examined the association between the levels of plasma calponin-h2 and clinicopathological parameters. The results demonstrated that the plasma level of calponin-h2 in breast cancer was significantly higher than those in the healthy control and benign breast disease groups $(\mathrm{P}<0.05)$. The combination of calponin-h2, carcinoembryonic antigen, carbohydrate antigen 15-3 improved the diagnosis of breast cancer. The plasma levels of calponin-h2 PR- breast cancers was significantly higher, compared with $\mathrm{PR}+$ breast cancers $(\mathrm{P}=0.033)$, and the plasma levels of calponin-h2 in patients with breast cancer aged $>50$ years was significantly higher than in patients $\leq 50$ years of age $(\mathrm{P}=0.001)$. No association was found between the level of plasma calponin-h2 and
\end{abstract}

Correspondence to: Mr. Qiang Zhou, Department of Clinical Laboratory, The Second Affiliated Hospital of Guangzhou Medical University, 250 Changgang Road, Haizhu Haizhuqu, Guangzhou, Guangdong 510260, P.R. China

E-mail: qiangzhou70@163.com

Mr. Xuguang Guo, Department of Clinical Laboratory, The Third Affiliated Hospital of Guangzhou Medical University, 63 Duobao Road, Guangzhou, Guangdong 510140, P.R. China

E-mail: gysygxg@gmail.com

Key words: calponin-h2, breast cancer, biomarker other clinicopathological parameters of breast cancer. Taken together, these results indicated that calponin-h2 may be a useful marker of breast cancer.

\section{Introduction}

Breast cancer is one of the most common types of malignancy and is the second leading cause of cancer-associated mortality among females, with an increasing incidence in several countries, including China $(1,2)$. Over the past few decades, novel techniques and methods for diagnosis and treatment have been developed, improving long-term survival rates of patients with breast cancer (3) Currently, clinical examination, ultrasound and mammography, plasma carcinoembryonic antigen (CEA) and carbohydrate antigen 15-3 (CA15-3) are the most widely applied techniques and parameters for auxiliary diagnosis, monitoring recurrence or metastasis in treated patients and predicting response or resistance to therapies. However, these methods either lack specificity, to a degree, or have poor positive predictive values $(4,5)$. Noninvasive and specific biomarkers for the early diagnosis of breast cancer remain an urgent requirement.

Nuclear matrix proteins (NMPs), the structural framework scaffolding of the nucleus, have been demonstrated to be involved in several vital cellular functions, including steroid hormone binding, DNA replication, gene transcription and translation. Due to the important roles of the nuclear matrix in these vital cellular functions, changes in the structure or components of NMPs may be implicated in the alteration in cellular and nuclear structure of cancer cells, and alterations of several NMPs have been demonstrated as cancer-specific biomarkers, as they can be detected at elevated levels in the plasma of cancer patients due to the release from dying cells in a soluble form (6). Therefore, plasma cancer-specific NMPs may be a candidate marker with improved specificity and sensitivity $(7,8)$.

Debald et al (9) identified five nuclear matrix proteins, which were upregulated in human breast cancer tissue, but were absent in healthy and benign controls. One of the breast cancer-specific proteins was confirmed as calponin-h2, a member of the calponin-h2 family. Calponin-h2 is expressed in smooth muscle and non-muscle cells, and is upregulated in 
growing smooth muscles, including the embryonic stomach, urinary bladder, and the uterus during early pregnancy in mice $(10,11)$. An appropriate level of h2-calponin may be critical to maintain the physiological function of different cells, including cell proliferation, remodeling, motility and phagocytosis (11-13). However, the association between plasma calponin-h2 and breast cancer remains to be elucidated. The aims of the present study were to investigate the plasma levels of calponin-h2 in patients with breast cancer, benign breast diseases and in healthy controls, to examine the association between plasma levels of calponin-h2 and clinicopathological parameters of breast cancer, and to compare the tissue levels of calponin-h2 levels between breast cancer and healthy controls.

\section{Patients and methods}

Patients. The population examined in the present study included 156 females, recruited from The Second Affiliated Hospital of Guangzhou Medical University (Guangzhou, China) between 2010 and 2012, comprising 100 patients with breast cancer, 30 patients with benign breast disease, and 26 healthy controls. All breast cancer and benign breast diseases underwent breast mass resection with histological confirmation of diagnosis. Patients with severe infection, active clinical comorbidities or a history of any other malignancy were excluded from the investigation. The healthy controls were recruited from the check-up center at The Second Hospital of Guanzhou Medical University and presented a negative mammogram result, indicating the absence of breast cancer, with no history of cancer, chronic disease or medications, excluding hormonal contraception. The present study was approved by the Guangzhou Medical University Ethics Committee (Guangzhou, China). Written informed consent was obtained from each patient and control individual prior to obtaining blood samples. The clinicopathological characteristics of the population are summarized in Table I.

Samples. Non-fasting venous blood samples $(4 \mathrm{ml})$ were collected from patients with breast cancer, benign breast disease and the healthy controls into heparin blood tubes. The blood samples were centrifuged at 1,610 x g for $10 \mathrm{~min}$ at room temperature and the plasma was aliquoted into micro-centrifuge tubes and stored at $-20^{\circ} \mathrm{C}$ until use. The blood samples from the patients with breast cancer and breast benign disease were obtained prior to surgery.

Enzyme-linked immunosorbent assay (ELISA) analysis. The plasma concentrations of calponin-h2 were determined using a commercially available quantitative kit (E98410HU; USCN Life Science, Inc., Wuhan, China), according to the manufacturer's instructions. Briefly, $100 \mu$ l each dilution of the standard, blank and samples (1:5 dilution) were incubated for $2 \mathrm{~h}$ at $37^{\circ} \mathrm{C}$. Subsequently, the medium of each well was replaced with detection reagent $\mathrm{A}(100 \mu \mathrm{l} /$ well $)$ and incubated for $1 \mathrm{~h}$ at $37^{\circ} \mathrm{C}$. Following removal of the medium and washing three times, $100 \mu \mathrm{l}$ detection reagent $\mathrm{B}$ was added, followed by incubation for $30 \mathrm{~min}$ at $37^{\circ} \mathrm{C}$. The wells were washed five times and $90 \mu \mathrm{l}$ substrate solution was added to each well and incubated for $15 \mathrm{~min}$ at $37^{\circ} \mathrm{C}$. Following incubation, $50 \mu \mathrm{l}$ stop solution was added to each well and the absorbance was read at $450 \mathrm{~nm}$ using a spectrophotometer (SLT Lab Instruments, Salzburg, Austria). The concentration of calponin-h2 in the plasma was interpolated from a standard curve, which was generated using the recombinant protein with the CurveExpert 1.3 software (http://www.curveexpert.net).

Chemiluminescent microparticle immunoassay. Plasma CEA and CA15-3, which regarded as useful tumor markers for breast cancer, were routinely assessed using a chemiluminescent microparticle immunoassay with a commercially available kit (ARCHITECT CEA; Abbott Laboratories, Abbot Park, IL USA). The cut-off values for CEA and CA 153, recommended by the manufacturer, were $5.0 \mathrm{ng} / \mathrm{ml}$ and $31.3 \mu / \mathrm{ml}$, respectively.

Immunohistochemical staining. Comparative analysis was performed on tissue samples from 12 cases of breast infiltrating ductal carcinoma and 12 normal breast tissues. The tissue samples $(\sim 1 \mathrm{x} 1 \mathrm{x} 0.3 \mathrm{~cm})$ were obtained from Clinical Pathology of The Second Affiliated Hospital of Guangzhou Medical University. The tissues were fixed in $10 \%$ neutral buffered formalin. Briefly, the samples were dehydrated through an ethanol series then the tissue was cleared using xylene (Tianjin Zhiyuan Chemical Reagent Co., Ltd., Tianjin, China). The tissue samples were then immersed in paraffin then embedded in a paraffin block. Sections $(4 \mu \mathrm{m})$ were prepared from paraffin-embedded samples using a microtome (Leica RM2135; Leica Microsystems GmbH, Wetzlar, Germany). The slides were then washed in xylene and rehydrated using an ethanol series. The slides were then rinsed for $5 \mathrm{~min}$ using phosphate-buffered saline (PBS). The samples were incubated for $10 \mathrm{~min}$ in $3 \% \mathrm{H}_{2} \mathrm{O}_{2}$ to block endogenous peroxidases, then, slides were rinsed for 5 min using PBS. Paraffin-embedded tissue sections were blocked with 5\% normal goat plasma for $30 \mathrm{~min}$ following deparaffinization, rehydration and quenching of endogenous peroxidase activity. The samples were incubated with monoclonal mouse anti-calponin-h2 primary antibody (1:400; cat. no. Hoooo1265-M01A; Abnova, Taiwan, China) at $4^{\circ} \mathrm{C}$ overnight and peroxidase-labeled polyclonal goat anti-mouse immunoglobulin (Ig)M $\kappa(1: 500$; cat. no. 074-1803; KPL, Gaithersburg, Maryland, USA) at room temperature for $1 \mathrm{~h}$. Diaminobenzidine (Beyotime Institute of Biotechnology, Nantong, China) was used as a chromogen. Slides were counterstained with hematoxylin (Beyotime Institute of Biotechnology), and the specimens were mounted on slides using Aquatex mounting solution (Merck, Darmstadt, Germany). The slides were evaluated under a light microscope (Olympus BH-2; Olympus, Tokyo, Japan), and the tissues were classified into two categories, 0 or 1 , corresponding to low levels and high levels of expression, respectively.

Statistical analysis. Statistical analysis was performed using SPSS 17.0 software (SPSS Inc., Chicago, IL,USA). Comparisons between the levels of CEA, CA15-3 and calponin-h2 in the blood were performed using a Mann-Whitney $U$ test, Kruskal-Wallis test or Wilcoxon signed rank test, when appropriate. The differences in the levels of calponin-h2 in the tissues of the breast cancer and healthy control samples were evaluated using a $\chi^{2}$ test or Fisher exact test. Receiver operator characteristic (ROC) curve analysis and calculation 
Table I. Clinicopathalogical parameters of patients with breast cancer.

Characteristic Number of patients $(\%)$

Age (years)
$\leq 50$
$>50$

$50(50)$

Histological type

$$
\begin{aligned}
& \text { IDC } \\
& \text { DCIS } \\
& \text { Other }
\end{aligned}
$$

Tumor size $(\mathrm{cm})$

$$
\begin{aligned}
& \leq 2 \\
& >2, \leq 5 \\
& >5
\end{aligned}
$$

Undetermined

Lymph node status

$$
\begin{aligned}
& \text { N0 } \\
& \text { N1 } \\
& \text { N2 } \\
& \text { N3 }
\end{aligned}
$$

Undetermined

Distant metastasis

$$
\text { M0 }
$$

M1

Undetermined

Stage

I/II

III/IV

Undetermined

Grade

G1

G2

G3

Undetermined

ER status

Negative

Positive

Undetermined

PR status

Negative

Positive

Undetermined

HER2 status

$$
0
$$

1

Undetermined

P53 status

Negative

Positive

Undetermined

EGFR status

Negative

Positive

Undetermined
54 (54)

$28(28)$

8 (8)

4 (4)

90 (90)

69 (69)

24 (24)

33 (33)

64 (64)

49 (49)

$52(52)$

46 (46)

16 (16)

Table I. Continued.

\begin{tabular}{lr}
\hline Characteristic & Number of patients $(\%)$ \\
\hline Ki-67 status & \\
Negative & $75(75)$ \\
Positive & $25(25)$ \\
Undetermined & $0(0)$ \\
BCL-2 status & \\
Negative & $38(38)$ \\
Positive & $43(43)$ \\
Undetermined & $19(19)$ \\
\hline
\end{tabular}

IDC, invasive ductal carcinoma; DCIS, ductal carcinoma in situ; ER, estrogen receptor; PR, progesterone receptor; HER2, human epidermal growth factor receptor-2; EGFR, epidermal growth factor receptor; BCL, B-cell lymphoma.

of the area under the curve (AUC) were performed in order to assess the ability of using plasma levels of CEA, CA15-3 and calponin-h2 to correctly distinguish between patients with breast cancer and healthy individuals, and patients with benign breast disease. An optimum diagnostic cut-off point for the population was selected to maximize the clinical sensitivity and specificity. The combination of CEA, CA15-3 and calponin-h2 were evaluated by logistic regression and ROC. $\mathrm{P}<0.05$ was considered to indicate a statistically significant difference.

\section{Results}

Increased plasma levels of calponin-h2 levels in patients with breast cancer. Comparison between the plasma levels of calponin-h2 in 26 healthy controls, 30 patients with benign breast disease and 100 patients with breast cancer were performed using ELISA. The median plasma levels of calponin-h2 in the healthy controls, patients with benign breast disease and patients with breast cancer were 12.81 (range 3.11-25.25), 10.35 (range 4.36-30.76) and $15.93 \mathrm{ng} / \mathrm{ml}$ (range 4.93-132.66 ng/ml) respectively. The plasma levels of calponin-h2 in the patients with breast cancer were significantly higher than those in the healthy control $(\mathrm{P}=0.016)$ and benign breast disease $(\mathrm{P}=0.001)$ groups. There were also significant differences in the plasma levels of CEA and CA15-3 between the patients with breast cancer and benign breast disease, and the controls $(\mathrm{P}<0.05)$. The median plasma levels of CEA and CA15-3 were $2.27 \mathrm{ng} / \mathrm{ml}$ (range 0.64-309.20 ng/ml) and $12.85 \mathrm{U} / \mathrm{ml}$ (range 4.30-97.70 U/ml) in the breast cancer group, $1.26 \mathrm{ng} / \mathrm{ml}$ (range $0.50-3.24 \mathrm{ng} / \mathrm{ml}$ ) and $10.55 \mathrm{U} / \mathrm{ml}$ (range 4.60-21.30 U/ml) in the benign breast disease group, $0.96 \mathrm{ng} / \mathrm{ml}$ (range 0.50-4.06) and $8.95 \mathrm{U} / \mathrm{ml}$ (range 4.70-26.3) in the healthy control group (Fig. 1).

Diagnostic value of plasma CEA, CA15-3 and calponin- $h 2$. To evaluate the diagnostic value of plasma levels of calponin-h2, CEA and CA15-3, the present study used ROC methods to calculate the sensitivity and specificity of plasma levels of calponin-h2, CEA and CA15-3. The AUC of the levels of 

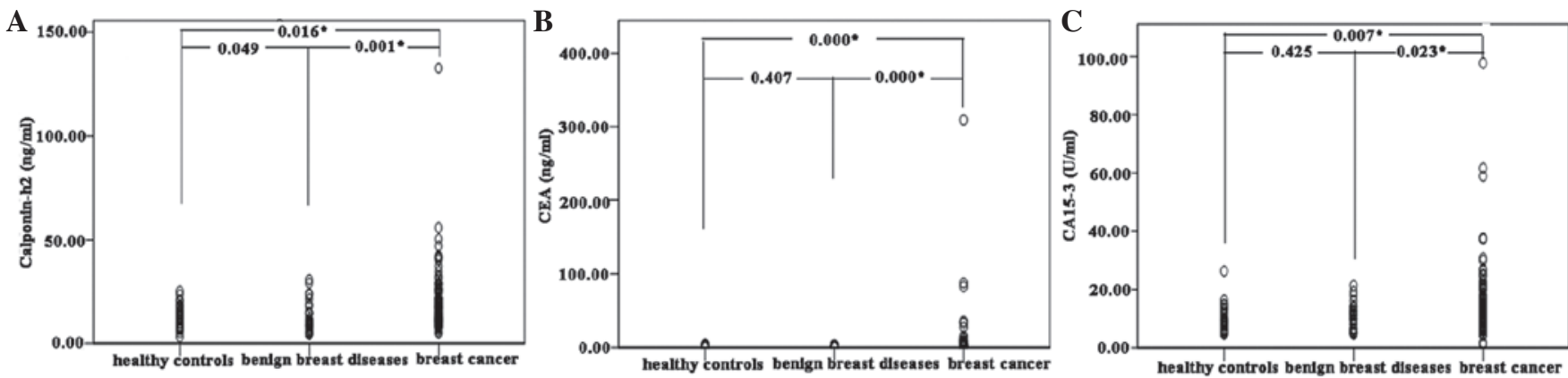

Figure 1. Plasma levels of calponin-h2, CEA, CA15-3 in normal controls, patients with benign breast disease and patients with breast cancer. (A) Plasma levels of calponin-h2 in patients with breast cancer were higher than those in the healthy controls and patients with benign breast disease. $(\mathrm{P}=0.016$ and $\mathrm{P}=0.001$, respectively; Mann-Whitney U test). (B) Plasma levels of CEA in patients with breast cancer were higher than those in the healthy controls and patients with benign breast disease ( $\mathrm{P}<0.001$; Mann-Whitny U test). (C) Plasma levels of CA15-3 in patients with breast cancer were higher than those in the healthy controls and patients with benign breast disease $(\mathrm{P}=0.007$ and $\mathrm{P}=0.023$, respectively; Mann-Whitney $\mathrm{U}$ test). $\mathrm{CEA}$, carcinoembryonic antigen; CA15-3, carbohydrate antigen 15-3.
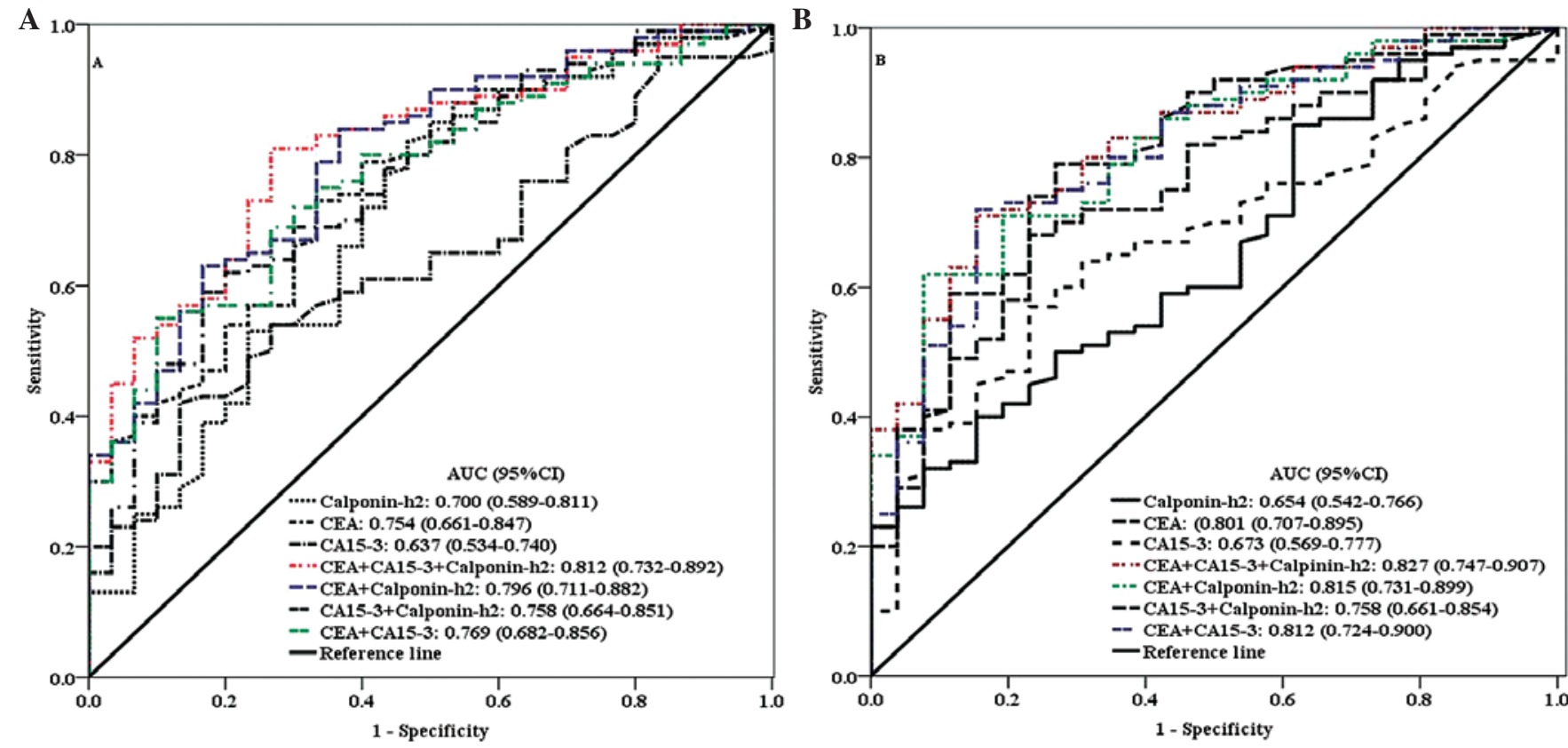

Figure 2. ROC curves of calponin-h2, CEA and CA15-3 were produced by plotting the association between specificity and sensitivity at various cut-off levels. (A) AUC of plasma levels of calponin-h2, CEA and CA15-3 in differentiating benign breast disease and breast cancer. (B) AUC of plasma levels of calponin-h2, CEA and CA15-3 in differentiating healthy control and breast cancer.

calponin-h2, CEA and CA15-3 in the blood from differentiating breast cancer and benign breast disease were 0.700 [95\% confidence interval (CI), 0.589-0.811], 0.754 (95\% CI, $0.661-0.847)$ and 0.637 (95\% CI, 0.534-0.740), respectively (Fig. 2). The optimal cut-off of point for the plasma level of calponin-h2 was $15.14 \mathrm{ng} / \mathrm{ml}$, based on maximization of the Yuden index, resulting in $53.0 \%$ sensitivity, $76.7 \%$ specificity, $69.4 \%$ positive predictive value (PPV), and $62.0 \%$ negative predictive value (NPV). Notably, the AUC of the combination of the three markers reached 0.812 (95\% CI, 0.732-0.892) and the AUC of the association between CEA and calponin-h2, CA15-3 and calponin-h2, and CEA and CA15-3 reached 0.796 (95\% CI, 0.711-0.882), 0.758 (95\% CI, 0.664-0.851) and 0.769 (95\% CI, 0.682-0.856), respectively (Fig. 2). Additionally, the AUC of the levels of calponin-h2, CEA and CA15-3 between patients with breast cancer and healthy controls were 0.654
(95\% CI, 0.542-0.766), 0.801 (95\% CI, 0.707-0.895) and 0.673 (95\% CI, 0.569-0.777), respectively (Fig. 3). The optimal cut-off of plasma calponin-h2 level was $10.04 \mathrm{ng} / \mathrm{ml}$, based on the maximization of Yuden index, resulting in $85.0 \%$ sensitivity, $39.5 \%$ specificity, $58.4 \%$ PPV, and $72.5 \%$ NPV. The AUC of the combination of the three markers reached 0.827 (95\% CI, 0.747-0.907), and the AUC of the combination of CEA and calponin-h2, CA15-3 and calponin-h2 and CEA and CA15-3 were 0.815 (95\% CI, 0.731-0.899), 0.758 (95\% CI, 0.661-0.854) and 0.812 (95\% CI, 0.724-0.900), respectively (Fig. 3). These data suggested that the combination of calponin-h2, CA15-3 and CEA may increase the diagnostic accuracy of breast cancer.

Association between plasma CEA, CA15-3 and calponin- $h 2$ and clinicopathological parameters. To examinee the 

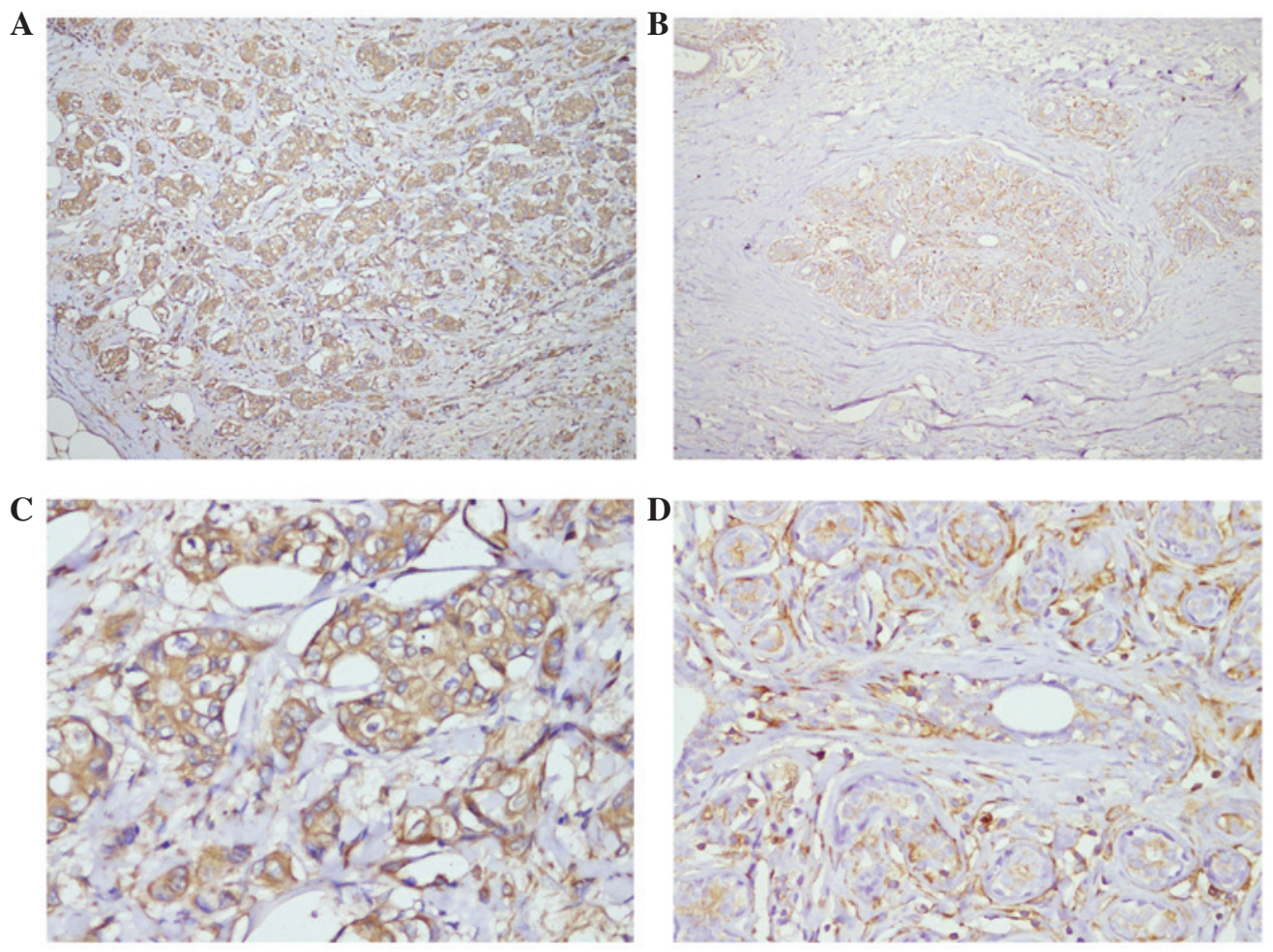

Figure 3. Immunohistochemical detection of calponin-h2 in (A) breast cancer tissue and (B) normal breast tissue. The expression of calponin-h2 in (A and C) breast cancer was higher, compared with that in the (B and D) healthy control. Calponin-h2 was primarily located in the cytoplasm. A and B: magnification, x100; C and D: magnification, x400. Nuclei were stained with hematoxylin.

potential prognostic roles of plasma calponin-h2 in breast cancer, the present study further analyzed the association between the levels of plasma calponin-h2 and the clinicopathological parameters of breast cancer. However, the plasma level of calponin-h2 was not associated with the tumour size, lymph node metastasis, distant metastasis, tumor-node-metastasis stage, endoplasmic reticulum, human epidermal growth factor (HER-2), Ki67, P53 or B-cell lymphoma 2. However, the plasma levels of calponin-h2 in PR- breast cancer was significantly higher, compared with $\mathrm{PR}+$ breast cancer $(\mathrm{P}=0.033)$, and plasma level of calponin-h2 levels in patients with breast cancer aged $>50$ years were significantly higher, compared with that in patients $\leq 50$ years old $(\mathrm{P}=0.001)$. The plasma level of CA15-3 in the HER-2+ breast cancer, was higher than that in HER-2- breast cancer $(\mathrm{P}=0.026)$. Plasma levels of CEA were also associated with the stages of breast cancer $(\mathrm{P}=0.035)$. Overall, these results indicated that plasma CEA and CA15-3 were not linked to the other clinicopathological parameters of breast cancer (Table II).

Upregulation of calponin- $h 2$ in human breast cancer tissues. The present study subsequently analyzed tissue expression levels of calponin-h2 in 12 normal breast epithelia and 12 invasive ductal carcinoma samples using immunohistochemistry. The results demonstrated that the breast cancer group exhibited a significantly higher rate of upregulated expression of calponin-h2, compared with the control group, at 66.7 $(8 / 12)$ and $16.7 \%(2 / 12)$, respectively $(\mathrm{P}=0.036$; Table III). It is noteworthy that calponin-h2 was located predominantly in the cytomembrane of the breast cancer tissues (Fig. 3).

\section{Discussion}

In the last few decades, the incidence of breast cancer has increased, however the relative survival rates of breast cancer have improved, due to early diagnosis and treatment of primary and second breast cancer (3). Early detection is vital to improve the prognosis of patients with cancer $(14,15)$. Although current breast cancer screening methods have contributed substantially to the reduction in breast cancer mortality rates, it is limited due to low specificity. Therefore, more specific non-invasive methods are urgently required, and plasma markers may offer a better alternative option.

Changes in the structure or components of NMPs may be an ideal marker for cancer diagnosis and prognosis $(8,16)$. Debald et al (9) found that the calponin-h2 NMP was upregulated in human breast cancer tissue and absent in healthy and benign control tissues. As NMPs can be released into plasma, the present study comparatively analyzed the plasma levels of calponin-h2 in patients with breast cancer, patients with benign breast disease and healthy controls, and found that plasma levels of calponin-h2 were higher in breast cancer than in benign breast disease, but not healthy controls. The AUC of the level of calponin-h2 of calponin-h2 levels in differentiating breast cancer and benign breast diseases $(0.700 ; 0.734-0.868 ; \mathrm{P}=0.0001)$ was higher than that of CA15-3, and lower than that of CEA (Fig. 2). It has been proposed that a combination of multiple markers improves the capacity to diagnose cancer, as single markers have a low capacity in the diagnosis of breast cancer. The present results demonstrated that a combination of the three markers was able to improve the diagnosis capacity. 
Table II. Association between the plasma levels of calponin-h2, CEA, CA15-3, and clinicopathological parameters of breast cancer.

\begin{tabular}{|c|c|c|c|c|c|c|}
\hline Characteristic & $\begin{array}{l}\text { Calponin-h2 } \\
\text { (ng/ml) }\end{array}$ & P-value & CA15-3 (U/ml) & P-value & CEA (ng/ml) & P-value \\
\hline Age (years) & & $0.001^{\mathrm{a}}$ & & 0.288 & & 0.226 \\
\hline$\leq 50$ & $12.05(4.93-55.78)$ & & $13.90(4.30-97.70)$ & & $1.98(0.73-309.20)$ & \\
\hline$>50$ & $19.67(6.45-132.66)$ & & $12.80(4.60-37.60)$ & & $2.62(0.64-35.56)$ & \\
\hline Histological type & & 0.972 & & 0.364 & & 0.137 \\
\hline IDC & $16.11(4.94-132.66)$ & & $13.80(4.30-97.70)$ & & $2.27(0.64-309.20)$ & \\
\hline DCIS & $14.77(7.84-42.41)$ & & $12.05(5.70-24.90)$ & & $2.08(1.06-9.44)$ & \\
\hline Others & $20.94(8.10-50.24)$ & & $12.80(6.00-26.50)$ & & $2.77(0.95-15.44)$ & \\
\hline Tumor size (cm) & & 0.426 & & 0.280 & & 0.156 \\
\hline$\leq 2$ & $17.43(6.45-132.66)$ & & $12.10(4.50-37.60)$ & & $2.26(0.64-15.44)$ & \\
\hline$>2, \leq 5$ & $15.30(4.93-46.95)$ & & $15.15(4.30-97.70)$ & & $2.77(0.78-309.20)$ & \\
\hline$>5$ & $14.38(11.00-21.50)$ & & $15.20(10.3-20.00)$ & & $2.48(2.11-4.89)$ & \\
\hline Lymph node status & & 0.265 & & 0.152 & & 0.194 \\
\hline N0 & $16.80(4.93-132.7)$ & & $12.80(4.30-97.70)$ & & $2.27(0.64-87.73)$ & \\
\hline N1 & $14.86(7.91-41.59)$ & & $15.15(4.50-58.90)$ & & $2.19(0.89-9.11)$ & \\
\hline $\mathrm{N} 2$ & $22.69(9.16-37.24)$ & & $9.60(6.00-16.60)$ & & $3.875(1.65-309.2)$ & \\
\hline N3 & $13.07(5.26-18.18)$ & & $15.00(6.00-37.50)$ & & $2.46(1.04-82.76)$ & \\
\hline Distant metastasis & & 0.711 & & 0.633 & & 0.068 \\
\hline M0 & $15.93(4.93-132.7)$ & & $13.00(4.50-61.60)$ & & $2.275(0.64-82.76)$ & \\
\hline M1 & $16.78(10.9-41.24)$ & & $15.90(4.30-97.70)$ & & $19.80(0.99-309.2)$ & \\
\hline Stage & & 0.690 & & 0.448 & & $0.035^{\mathrm{a}}$ \\
\hline $\mathrm{I} / \mathrm{II}$ & $15.74(4.93-132.66)$ & & $13.10(4.50-61.60)$ & & $2.26(0.64-32.90)$ & \\
\hline III/IV & $15.49(5.26-41.24)$ & & $12.60(4.30-97.70)$ & & $2.88(0.99-309.20)$ & \\
\hline Grades & & 0.222 & & 0.510 & & 0.955 \\
\hline G1 & $18.38(10.61-46.95)$ & & $11.10(5.70-21.00)$ & & $2.18(1.31-9.44)$ & \\
\hline $\mathrm{G} 2$ & $12.90(4.93-132.65)$ & & $13.70(4.50-97.70)$ & & $2.30(0.64-309.2)$ & \\
\hline G3 & $17.30(6.45-55.78)$ & & $12.90(4.30-37.60)$ & & $2.28(0.78-35.56)$ & \\
\hline ER status & & 0.201 & & 0.591 & & 0.918 \\
\hline Negative & $17.43(6.45-41.59)$ & & $12.80(4.30-58.90)$ & & $2.35(0.84-309.20)$ & \\
\hline Positive & $14.36(4.93-132.66)$ & & $13.20(4.50-97.70)$ & & $2.28(0.64-87.73)$ & \\
\hline PR status & & $0.033^{\mathrm{a}}$ & & 0.429 & & 0.928 \\
\hline Negative & $17.43(6.45-55.78)$ & & $12.80(4.30-58.90)$ & & $2.30(0.78-35.56)$ & \\
\hline Positive & $12.75(4.93-132.66)$ & & $13.45(4.50-97.70)$ & & $2.28(0.64-309.2)$ & \\
\hline HER-2 status & & 0.696 & & $0.026^{\mathrm{a}}$ & & 0.621 \\
\hline 0 & $16.11(7.25-132.66)$ & & $12.6(4.30-97.70)$ & & $2.27(0.64-87.73)$ & \\
\hline 1 & $15.56(4.93-55.78)$ & & $15.65(5.70-61.60)$ & & $2.44(0.95-309.2)$ & \\
\hline P53 status & & 0.656 & & 0.172 & & 0.850 \\
\hline Negative & $16.22(7.25-55.78)$ & & $12.80(4.30-97.70)$ & & $2.26(0.64-309.20)$ & \\
\hline Positive & $15.56(4.93-132.66)$ & & $14.45(4.60-61.60)$ & & $2.33(0.73-82.76)$ & \\
\hline EGFR status & & 0.717 & & 0.376 & & 0.609 \\
\hline Negative & $15.38(4.93-132.66)$ & & $13.70(4.5-97.7)$ & & $2.51(0.64-309.20)$ & \\
\hline Positive & $17.43(6.45-41.24)$ & & $13.10(4.3-24.8)$ & & $2.61(0.99-7.46)$ & \\
\hline Ki67 status & & 0.167 & & 0.471 & & 0.366 \\
\hline Negative & $14.88(5.26-132.66)$ & & $12.80(4.30-97.70)$ & & $2.26(0.64-309.20)$ & \\
\hline Positive & $17.65(4.93-55.78)$ & & $14.80(6.00-61.60)$ & & $2.57(0.78-35.56)$ & \\
\hline BCL2 status & & 0.443 & & 0.247 & & 0.095 \\
\hline Negative & $16.77(6.45-132.66)$ & & $12.85(4.3-58.9)$ & & $2.9(0.89-82.76)$ & \\
\hline Positive & $15.22(4.93-46.95)$ & & $13.8(5.6-61.6)$ & & $2.225(0.64-309.2)$ & \\
\hline
\end{tabular}

Data are expressed as the median (range). ${ }^{\mathrm{a}} \mathrm{P}<0.05$, plasma calponin- $\mathrm{h} 2$ level between breast cancer patients $\leq 50$ years and breast cancer patients >50 years. IDC, invasive ductal carcinoma; DCIS, ductal carcinoma in situ; ER, estrogen receptor; PR, progesterone receptor; HER2, human epidermal growth factor receptor-2; EGFR, epidermal growth factor receptor; BCL, B-cell lymphoma. 
Table III. Proportion of low and high expression levels of calponin-h2 in breast cancer and healthy control tissue samples.

\begin{tabular}{lcccr}
\hline & & \multicolumn{2}{c}{ Expression level of calponin-h2 [n (\%)] } \\
Group & Sample (n) & Low (\%) & High (\%) & P-value \\
\hline Invasive ductal carcinoma & 12 & $4(33.3)$ & $8(66.7)$ & $0.036^{\mathrm{a}}$ \\
Healthy control & 12 & $10(83.3)$ & $2(16.7)$ & \\
\hline
\end{tabular}

${ }^{\mathrm{a}}<0.05$

The present study also analyzed the association between plasma levels of CEA, CA15-3 and calponin-h2, and the clinicopathological parameters of breast cancer. The plasma level of calponin-h2 was not associated with tumor stage or grade, tumor size, lymph and distant metastasis, ER, HER-2, Ki-67 or P53 (Table II). Notably, the plasma level of calponin-h2 was associated with the expression of PR and the age of the patient with breast cancer. The present study also demonstrated a the positive correlation between plasma CEA and the stage of breast cancer. Previous larger population studies indicated that higher preoperative levels of CA15-3 and CEA were significantly associated with a larger tumor size, axillary node metastases and advanced stage (17). The difference in these results to those of the present study may be linked to the smaller sample size in the present study. Therefore, the roles of plasma calponin-h2 in breast cancer diagnosis and its association with the clinicopathological parameters of breast cancer require further investigation.

The present study also compared the expression of calponin-h2 in breast cancer tissue and normal breastepithelium using immunohistochemistry, and found that calponin-h2 was significantly overexpressed in breast cancer tissue, compared with normal breast tissues, particularly in the cytomembrane of the breast cancer cells. Debald M et al (9) reported, via one-dimensional immuniblotting, that calponin-h2 is expressed in the NMP- fraction of histologically different human breast cancer entities (ductal, lobular and mucinous) and breast cancer lines, but not in the NMP- fraction of healthy human breast tissue and cytoplasmic proteins of breast cancer. Congruously, the present study revealed no expression of calponin-h2 in the cytoplasmic fraction of human breast cancer tissues. Calponin-h2 is also overexpressed in human rectal carcinoma and cutaneous squamous cell carcinoma, indicating that the overexpression of calponin- $\mathrm{h} 2$ is involved in the pathogenesis of several types of cancer, although its function remains to be elucidated $(18,19)$.

In conclusion, the results of the present study indicated that calponin-h2 was upregulated in the tissue and plasma of patients with breast cancer, and may be a promising marker of breast cancer. The involvement and underlying mechanisms of calponin-h2 in carcinogenesis require further investigation.

\section{Acknowledgements}

The authors would like to thank all the patients involved. This study was partially supported by the Guangzhou Medical College Science Foundation Program (grant. no. 2012A08).

\section{References}

1. Benson JR and Jatoi I: The global breast cancer burden. Future Oncol 8: 697-702, 2012.

2. Chen WQ, Zhang SW, Zou XN and Zhao P: Cancer incidence and mortality in china, 2006. Chin J Cancer Res 23: 3-9, 2011.

3. Coleman MP, Forman D, Bryant $\mathrm{H}$, et al: Cancer survival in Australia, Canada, Denmark, Norway, Sweden and the UK, 1995-2007 (the International Cancer Benchmarking Partnership): an analysis of population-based cancer registry data. Lancet 377 : 127-138, 2011.

4. Chérel P, Hagay C, Benaim B, et al: Mammographic evaluation of dense breasts: techniques and limits. J Radiol 89: 1156-1168, 2008 (In French).

5. Safi F, Kohler I, Rottinger E, Suhr P and Beger HG: Comparison of CA15-3 and CEA in diagnosis and monitoring of breast cancer. Int J Biol Markers 4: 207-214, 1989.

6. Miller TE, Beausang LA, Winchell LF and Lidgard GP: Detection of nuclear matrix proteins in serum from cancer patients. Cancer Res 52: 422-427, 1992.

7. Leman ES and Getzenberg RH: Nuclear structure as a source of cancer specific biomarkers. J Cell Biochem 104: 1988-1993, 2008.

8. Higgins G, Roper KM, Watson IJ, et al: Variant Ciz1 is a circulating biomarker for early-stage lung cancer. Proc Natl Acad Sci USA 109: E3128-E3135, 2012.

9. Debald M, Franken S, Heukamp LC, et al: Identification of specific nuclear structural protein alterations in human breast cancer. J Cell Biochem 112: 3176-3184, 2011.

10. Wu KC and Jin JP: Calponin in non-muscle cells. Cell Biochem Biophys 52: 139-148, 2008.

11. Hossain MM, Hwang DY, Huang QQ, Sasaki Y and Jin JP: Developmentally regulated expression of calponin isoforms and the effect of h2-calponin on cell proliferation. Am J Physiol Cell Physiol 284: C156-C167, 2003.

12. Huang QQ, Hossain MM, Wu K, Parai K, Pope RM and Jin JP: Role of H2-calponin in regulating macrophage motility and phagocytosis. J Biol Chem 283: 25887-25899, 2008.

13. Ulmer B, Hagenlocher C, Schmalholz S, et al: Calponin 2 acts as an effector of noncanonical Wnt-mediated cell polarization during neural crest cell migration. Cell Rep 3: 615-621, 2013.

14. Walters S, Maringe C, Butler J, et al: Breast cancer survival and stage at diagnosis in Australia, Canada, Denmark, Norway, Sweden and the UK, 2000-2007: a population-based study. Br J Cancer 108: 1195-1208, 2013.

15. Houssami N, Ciatto S, Martinelli F, Bonardi R and Duffy SW: Early detection of second breast cancers improves prognosis in breast cancer survivors. Ann Oncol 20: 1505-1510, 2009.

16. Santoni M, Catanzariti F, Minardi D, et al: Pathogenic and Diagnostic Potential of BLCA-1 and BLCA-4 Nuclear Proteins in Urothelial Cell Carcinoma of Human Bladder. Adv Urol 2012: 397412, 2012.

17. Lee JS, Park S, Park JM, Cho JH, Kim SI and Park BW: Elevated levels of preoperative CA15-3 and CEA serum levels have independently poor prognostic significance in breast cancer. Ann Oncol 24: 1225-1231, 2013.

18. Choi SY, Jang JH and Kim KR: Analysis of differentially expressed genes in human rectal carcinoma using suppression subtractive hybridization. Clin Exp Med 11: 219-226, 2011.

19. Dang C, Gottschling M, Manning K, et al: Identification of dysregulated genes in cutaneous squamous cell carcinoma. Oncol Rep 16: 513-519, 2006. 